\title{
DIGNITY-ENHANCING NURSING CARE
}

\section{A FOUNDATIONAL ETHICAL FRAMEWORK}

\section{Chris GASTMANS}

\begin{abstract}
Starting from two observations regarding nursing ethics research in the past two decades namely, the dominant influence of both empirical methods and the principles approach - we present the cornerstones of a foundational argument-based nursing ethics framework. First, we briefly outline the general philosophical-ethical background from which we develop our framework. This is based on three aspects: lived experience, interpretative dialogue, and normative standard. Against this background, we identify and explore three key concepts vulnerability, care, and dignity - that must be observed in an ethical approach to nursing. Based on these concepts, we argue that the ethical essence of nursing is the provision of care in response to the vulnerability of a human being in order to maintain, protect, and promote his or her dignity as much as possible.
\end{abstract}

Keywords: nursing, ethics, lived experience, vulnerability, care, dignity 


\section{INTRODUCTION}

Since the beginning of the 1980s, nursing ethics has developed to such a degree that it is now considered a standard discipline within applied ethics. Nursing had to wait a long time for its ethical perspective to be recognized. This was due to many factors, including the almost exclusive focus on the 'act of medical decision-making' in traditional healthcare ethics, which led to a relative neglect of the wider context in which care decisions are made primarily by nurses. The $20^{\text {th }}$ anniversary of Nursing Ethics is an excellent moment to critically reflect on the philosophical foundations of nursing ethics as a scientific discipline.

Looking back to the nursing ethics literature published in the last two decades, two observations can be made. The first observation concerns the 'empirical turn' in healthcare ethics that seems to be most noticeable in the field of nursing ethics. A retrospective quantitative study of nine peer reviewed journals in the field of healthcare ethics revealed that the highest percentage of empirical research articles published between 1990 and 2003 appeared in Nursing Ethics (39.5\%), followed by the Journal of Medical Ethics (16.8\%) and the Journal of Clinical Ethics (15.4\%). ${ }^{1}$ What does this reveal about nursing ethics as an ethical discipline? Does the strong empirical embeddedness of nursing ethics imply that the philosophical-ethical or argument-based foundations of nursing ethics are set aside as less important by nursing ethics scholars? Whereas medical ethics scholars show a strong investment in foundational philosophical approaches to medical ethics.

The second observation is that the argument-based nursing ethics literature published in the last two decades is, in large measure, comprised by the four principles of biomedical ethics respect for autonomy, nonmaleficence, beneficence and justice. ${ }^{2}$ An illustrative example for this observation is the nursing ethics literature on euthanasia. It is striking that in most of the 41 argument-based nursing ethics articles on euthanasia we identified in a literature review ${ }^{3}$, 
principle-based arguments were addressed to a considerable extent. Especially the principles of respect for autonomy and non-maleficence were used in order to argue for and against euthanasia respectively. From another argument-based literature review ${ }^{4}$, we learned that the moral reasoning on intimacy and sexuality in institutionalized elderly persons was largely covered by the four principles of biomedical ethics. Again, the principle of respect for autonomy and the concomitant notion of informed consent were guiding the ethical debate. Arguments related to care (e.g. dignity, vulnerability, intersubjectivity) received considerably less attention. The dominant influence of the principles approach - and of the principle of respect for autonomy in particular - reflects a real challenge regarding the philosophical foundation of nursing ethics research. Is nursing still searching for its own ethical framework that can be distinguished from the principles approach?

Starting from these two observations, we present the cornerstones of a foundational argumentbased nursing ethics framework. First, we briefly outline the general philosophical-ethical background from which we develop our framework. Against this background, we identify three key concepts - vulnerability, care, and dignity - that express the essence of an ethical approach on nursing practice. With this ethical framework, we try to contribute to one of the essential tasks of nursing ethics, namely to reflect critically and systematically on nursing practices from the perspective of the good for all people involved.

\section{FROM PRINCIPLES TO DIGNITY-ENHANCING CARE}

\section{Four principles}

From the viewpoint of principlism - the dominant model in medical ethics - an ethical problem can be considered to be one of rights and duties. These rights and duties can be expressed at a theoretical level as conflicting principles, namely respect for autonomy, nonmaleficence, beneficence, and justice. ${ }^{2}$ These theoretical principles form the basis for 
finding a solution to clinical-ethical problems and for determining what is or is not relevant from an ethical point of view. Principlism exhibits all the characteristics of an ethical spirit of abstraction that focuses on identifying, categorizing and solving problems by generalizing the situation at hand. For instance, in the nursing ethics literature, euthanasia as an ethically good practice is often justified on the basis of respect for an individual's autonomy. A person requesting euthanasia organizes his or her own private life in such a way that doctors and nurses cannot counteract what he or she considers as the individual realization of his or her autonomous choice. ${ }^{3}$ The principle of respect for autonomy is also frequently mentioned in the ethics literature on sexuality in institutionalized elderly persons. It seems to be a predominating factor in assessing the moral permissibility of sexual behavior in institutionalized elderly persons. ${ }^{4}$

Although the four principles of biomedical ethics seem to play a prominent role in evaluating nursing practices, some authors are doubtful about an approach that focuses solely on these principles. As Moody pointed out, an important feature of the principles approach is its timelimited or action-focused quality. ${ }^{5}$ The central question is always: 'What is to be done?', that is, 'What act or decision is to be taken, under what intentions, and with what foreseeable consequences?' The primary focus is a specific, delimited act or choice (e.g. act of euthanasia, sexual act), not for example, a dynamic process of care or questions about someone's attitudes. The ethical dilemmas that nurses face cannot be contained within a few isolated decisions made in a single moment of time. Nurses caring for patients go through a whole process of care, during which they, in close interactions with patients, their relatives and physicians, continually have to make minor and major decisions linked to daily life issues such as hygiene, eating and drinking, intimacy and sexuality, etc. It is the whole history of the care process and its embeddedness in patients' personal life story, and the relationships between all involved in the care process, that is crucial. For instance, being involved in the 
care process for patients requesting euthanasia ${ }^{6}$ or dealing with expressions of sexual behavior of demented residents ${ }^{4}$ requires more than taking the right decision on a certain moment; it implies a continuous involvement of nurses with all their cognitive, attitudinal, communicative and interpretative capacities. The ethical quality of this integral care practice should be the focus of a nursing ethics approach. Hence, in order to deal adequately with ethical issues in nursing care, a wider ethical perspective is needed. We consider the following aspects to be essential in this respect (Figure 1): (1) lived experience, (2) interpretative dialogue, and (3) normative standard.

\section{(FIGURE 1)}

\section{Lived experiences}

The approach that we adopt is committed to the view that concrete lived experiences (e.g., of caregiving, care receiving, vulnerability, dignity) rather than abstract principles (e.g., respect for autonomy), should be the primary guide for developing a nursing ethics framework. Instead of trying to improve the ethical quality of nursing care practices on the basis of an external framework of normative principles to be applied to these practices, our approach is embedded in the practice of care itself. Intuitions or subjective feelings and ideas about care practices must indeed be clarified, as they have an illuminative character regarding the phenomenon of nursing care as it is experienced in everyday life. For instance, when we inquired Belgian nurses in a qualitative study about the way in which they experience their involvement in the euthanasia care process, all nurses described it as a grave and difficult process, not only on an organizational and practical level, but also on an emotional level. "Intense" was the dominant feeling experienced by nurses. ${ }^{6}$ These experiences should be taken into account in an ethical interpretation of nursing care for patients requesting euthanasia. Giving priority to concrete care experiences, which of course are not only those 
being shared by nurses, but also by patients, relatives, and others belonging to the care process, reveals that the phenomenon of nursing care and the ethical problems associated with it are far more complex than can be captured in a mere theoretical approach. Promoting this view, we claim that nursing ethics can benefit from a better understanding of care experiences as a whole and the rich and complex context within which they are situated.

\section{Interpretative dialogue}

Promoting the good of the patient by providing good care can be considered as a general aim of nursing. An important fact connected with nursing care is the dialogical context in which the search for the good takes place. There are always several parties involved in nursing care practices. Besides the patient and their relatives, there is the team of caregivers, usually of an interdisciplinary composition. It is necessary that all those involved in care processes are motivated to jointly explore the possible alternatives. For instance, in order to clarify a euthanasia request, nurses communicate often and a lot, with various people, in different contexts, in different ways and with various purposes. ${ }^{7}$ The experiences and standpoints expressed by all the people involved outline the rich narrative context within which concrete care processes take place.

If we are to consider seriously the dialogical aspect of nursing-ethical decision-making, it automatically follows that decision-making processes also have an interpretative aspect. The analysis of a problem from an interpretative perspective is characterized by the large amount of attention paid to interpreting the viewpoints of those involved with the ethical problem. One assumes that these viewpoints are never completely clear to those concerned. Even the person who voices a certain opinion is never totally aware of the complete contents, meanings, and consequences of his or her opinion. What a person exactly wants is never really clear. Sometimes, patients are even 'ambivalent' about treatment decisions, especially when 
confronted with life-threatening situations. ${ }^{8}$ Hence, viewpoints expressed by the people involved need to be gradually developed and interpreted.

\section{Normative standard}

An ethical approach is always in some way linked to the issue of normativity. The question of normativity in healthcare refers to two intrinsically interwoven groups of questions ${ }^{9}$ : One on the obligatory character of care (Why do we feel as if we should care?) and one on what makes care ethically sound (What counts as good nursing care?). Both groups of normative questions that guide our approach invoke a certain view of mankind that underlies care, that is, a specific anthropological framework. ${ }^{10}$ Only when the objective normative basis of nursing care is sufficiently clarified, care practices can be evaluated and optimized from an ethical point of view. To this end, the nature of the person performing nursing care - de nurse - and the nature of the person receiving care - the patient - should be sufficiently clear. Hence, an ethical approach to nursing should intend to deepen the normative value of nursing care by referring to its anthropological foundations.

\section{NURSING CARE CONSIDERED AS DIGNITY-ENHANCING CARE}

The three above mentioned aspects of our ethical framework inspire our reflection on the ethical essence of nursing. We argue that persons who are in need of nursing care are vulnerable human beings. This vulnerability shapes the nursing care process from the beginning and transforms it into an ethical process. In nursing care, it becomes clear that ethics arises from the appeal to be susceptible to the vulnerability of the person who is in need of care. Essentially, nursing care aims to lessen the vulnerability of a fellow human being or to deal with it in an appropriate way. As vulnerability is an essential component of nursing care processes, these care processes should always meet an ethical standard: one should respect the dignity of the vulnerable patient. Good nursing care aims at the enhancement of 
the dignity of the human person in all his or her dimensions and also succeeds to realize this intention in practice. Hence, good nursing care is to be considered as dignity-enhancing care.

Based on the above-mentioned normative characterization of nursing care, the ethical essence of nursing care can be defined as: providing care in response to the vulnerability of a human being in order to maintain, protect, and promote his or her dignity as much as possible. In the following sections, the meaning of vulnerability, care, and dignity — and thus of dignityenhancing care - will be explained in the context of nursing care. It will become clear that vulnerability, care, and dignity fully correspond with the three aspects — lived experience, interpretative dialogue, and normative standard, respectively — of our ethical framework (Figure 1).

\section{Vulnerability: a profound lived experience}

Since vulnerability is an essential part of the human condition, it is closely connected to our lived experience as human beings. As Robert Goodin argues, harm may come from many sources and we are never entirely free from the possibility of being harmed. ${ }^{11}$ But besides the ordinary human vulnerability we all share, there are people who are extraordinary vulnerable. ${ }^{12}$ More particularly, the experience of dementia produces such an extraordinary vulnerability. The kind of vulnerability that persons with dementia are confronted with is irreversible. Their vulnerability is situated in all dimensions of their being. Persons with dementia are not only vulnerable with respect to their frail bodies, but also in regard of the psychological, relational, social, moral, and spiritual dimensions of their being human, regardless of whether they are cognitively aware of their vulnerability. Moreover, their vulnerability is so overwhelming that it threatens the dignity of these persons.

Vulnerability and care are closely intertwined. Moreover, the essence of care could be summarized as 'responding to vulnerabilty'. The confrontation with forms of vulnerability 
motivates nurses to respond adequately and appropriately to the needs of the patient. For instance, when we interviewed Belgian nurses about the way in which they experience their involvement in the decision-making process concerning tube feeding in patients with dementia, the nurses reported it is precisely the vulnerable dependency of the patient that shaped a strong but often wordless appeal to them to provide dignified care. ${ }^{13}$ Nurses feel themselves touched not only as professionals, but also as persons. Due to their strong commitment to the patient, nurses experience their care for vulnerable patients as a moral duty. Moreover, it is exactly with persons whose rational capacities are minimal and whose corporal vulnerability is the greatest — like persons with severe dementia — that care appears to be the way in which another person connects himself or herself to them as a person and treats them as a person. ${ }^{10,13}$ Hence, vulnerability is not only connected with care, but also with ethics. Goodin states that the vulnerability of other human beings is the source of our moral responsibility to them. ${ }^{11}$ Ethics manifests itself par excellence in situations where a person's dignity is threatened due to his or her vulnerable situation, and where the person is unable to force a respectful attitude from the fellow human being. Applied to healthcare, as vulnerable patients cannot meet their own needs, they must rely on the goodwill of caregivers, like nurses. Power imbalances in nurse-patient relationships contribute to patients' vulnerability and their reliance on nurses' goodwill not to harm them. ${ }^{14}$ Hence, the practice of nursing includes a moral dimension that requires responsibility, sensitivity and trustworthiness. ${ }^{14}$ Geriatric nurses are frequently confronted with these kind of ethically sensitive situations, e.g. when dealing with bodily privacy during hygienic care procedures or with feelings of shame of older residents with swallowing problems during mealtime care. Such kind of situations easily lead to 'abuse' of the older person. 'Abuse' means in this context that nurses reduce their residents to their status of passive receivers of technical nursing care without taking into 
account the vulnerability as it is experienced by the older people themselves (e.g. feelings of shame as a result of loss of privacy or the incapacity to eat autonomously).

\section{Care: a dialogical-interpretative process}

Vulnerable people are in need of care. Margaret Walker characterizes care as a practice of responsibility, in which the various persons involved take responsibility in a process of reacting to vulnerability. ${ }^{15}$ It is the situation of vulnerability of the fellow human being that prompts us to care for the other. In this way, care starts from the appeal to be susceptible to the lot of other people in an attentive, responsible, competent and responsive way.

An important fact connected with nursing care is the dialogical context in which nursing care practices are situated. By providing nursing care, and the attitudes and skills associated with this activity, nurses enter as a person into a relationship with a vulnerable fellow human being who is in need of care. Concern about the vulnerable state in which a patient finds himself or herself is the point of departure of nursing care. Belgian nurses caring for hospitalized patients with dementia considering artificial nutrition and hydration revealed that they put themselves in the patient's place and tried to imagine the patients' experiences. Empathy enabled nurses to develop great concern about the well-being of the patient. ${ }^{13}$ Tronto referred in this respect to the ethical attitude of attentiveness. ${ }^{16}$ Attentive nurses take up a receptive position with respect to the patient: They are challenged to step out of their own personal framework in order to take up that of the patient, so that they can better understand his or her real-life situation. Without an attitude of attentiveness the request for care will not even be noticed.

However, it is not clear from the beginning what answer can be considered as the most adequate and appropriate answer to the care needs of a particular patient. Finding the right answer is not the result of a general and abstract balancing of principles or of logical deduction. On the contrary, it is reached through a shared dialogical process of 
communication, interpretation, and understanding that takes place within the care relationship. It is precisely in this relational context — through a process of choice and deliberation — that goals and appropriate means for providing nursing care are set up. In the above mentioned example of decision-making regarding artificial nutrition and hydration, nurses and physicians are searching through dialogue and deliberation for answers to the question whether artificial nutrition and hydration can contribute to the patient's good and dignity. ${ }^{13}$ Nursing care practices are characterized by the unique capacity to make choices in particular situations that bring about more dignity for vulnerable fellow human beings. ${ }^{17}$ Responsibility and competency are the two ethical attitudes that are needed to find a good answer to the vulnerability of fellow human beings. ${ }^{16}$

Nursing care consists of the needs and wants of the patient and the responsibility and competency that the nurse adopts to meet those needs. However, the patient is not just a passive partner in the care process. Ethically sound nursing care exists when it is properly provided and received. Nursing care demands feedback and the verification that caring needs are actually being met. Thus, reciprocity is in principle an important part of nursing care. Patients show respect to their nurses by allowing themselves to be cared for and by responding appropriately to the care; for example, by expressing gratitude or by expressing displeasure. For this reason, Tronto considered 'care receiving' to be an essential dimension in the care process, linked to the attitude of responsiveness. ${ }^{16}$ However, the meaningfulness of the care provided should in no way be made dependent on the patient's capacity to respond. Caring for persons who have less or no exchange power (e.g. a persistent vegetative state patient or even a dead patient) should not a priory be considered less worthy. Nurses should provide services with respect for human dignity of the patient unrestricted by considerations of the status of the patient or the nature of his or her health problems.

\section{Dignity: a normative standard}


One of the key messages we emphasized throughout the previous paragraphs is that nursing care that is provided to vulnerable persons is expected to be 'good care' in the ethical meaning of the word. But what does good nursing care mean? We assume, for instance, that good nursing care deals with all kinds of vulnerabilities patients are confronted with. This is care that, given the vulnerable status of the patient, supports the dignity of the human person as much as possible. We use the concept of 'dignity-enhancing care' to name this kind of care. By using the concept of 'dignity-enhancing care', we are inspired by Harvey Max Chochinov, who uses the concept of 'dignity-conserving care'. ${ }^{18}$ In order to clarify this normative concept, we should further clarify the inherent ethical meaning of nursing care.

When we look at nursing care from an ethical perspective, noteworthy is the goal-oriented character of nursing care. Whatever nurses do must always be related to the final goal that is set. Generally, the goal of nursing care is described as the promotion of the dignity of the patient by providing good care in the wider meaning of the word - i.e., on the physical as well as the psychological, relational, social, moral, and spiritual level. As nursing care is directed toward the promotion of the dignity of the patient by providing good care — the myth of the neutrality of the caring process is radically questioned. Nursing care can be considered to be a moral practice. ${ }^{17}$ The ethical concern for the patient's well-being and dignity, which is mainly based on respect for the person in his or her totality, is fundamental to the moral demand that inspires nursing care. In each particular situation, the patient, together with his or her relatives and nurses, searches for appropriate means to achieve as much good as possible. Hence, filling in the content of dignity-enhancing care is of essential importance for the ethical evaluation of nursing care.

It is beyond the scope of this article to formulate a full description of a normative concept such as dignity-enhancing care. In nursing care, the bodily aspects often come first, because generally they are most easily translated into complaints for nurses to address. However, a 
nurse who intends to approach the patient as a whole pays attention not only to the physical aspects, but also to the relational, social, psychological, ethical, and spiritual dimensions of being a person. The vulnerability that affects the patient in all these dimensions results in the dignity of the person being threatened. Dignity-enhancing nursing care aims to respond to all these aspects of human vulnerability in order to enhance the dignity of the person as much as possible. This brings us to the premise that our ethical approach to nursing should be closely related to the notion of personhood. The ethical reflection on care practices always starts from the assumption of a certain view on the human person. When this view on the human person is made explicit, what is understood by 'dignity-enhancing care' can be clarified. Elsewhere, we made our view on the human person explicit by linking it to the anthropology of Louvain personalism. ${ }^{10,17}$ Based on these fundamental explorations, we can state in general that the care for the patient is most meaningful when the patient is respected as a human person in all his or her dimensions: namely being related to the whole of reality, being embodied, being related to others, being related to the material world, being related to one's own history, being a product of one's culture, and being a unique and autonomous subject. This general demand of dignity-enhancing nursing care may help us avoid falling into one-sidedness. It appears to us to be easy to stress one or another aspect (e.g., bodily, psychological, spiritual) of nursing care. Such a 'reductionism' contains a real danger to dignity-enhancing care that aims to approach the patient as an integral person. In other words, in order to determine whether a nursing act, attitude or instrument is morally good, one must apply the criterion of dignity of the human person, considered in all his or her dimensions.

\section{CONCLUSION}

In this paper we presented an ethical approach to nursing care, taking into account three essential aspects: lived experience, interpretative dialogue, and normative standard. This framework, linked to three core concepts in nursing care — vulnerability, care, and dignity — 
enabled us to describe the general contours of a nursing ethics framework that is deeply anchored in essential aspects of nursing practice itself. However, more philosophical reflection is still needed in order to provide nursing with an ethical framework that can be considered to be a complementary alternative to the principles approach.

\section{REFERENCES}

(1) Borry P, Schotsmans P, Dierickx K. Empirical research in bioethical journals: a quantitative analysis. J Med Ethics 2006; 32:240-245.

(2) Beauchamp J and Childress J. Principles of biomedical ethics. $6^{\text {th }}$ edn. Oxford: Oxford University Press, 2009.

(3) Quaghebeur T, Dierckx de Casterlé B, Gastmans C. Nursing and euthanasia: a review of argument-based ethics literature. Nurs Ethics 2009; 16:466-486.

(4) Mahieu L and Gastmans C. Sexuality in institutionalized elderly: a systematic review of argument-based literature. Int Psychogeriatr 2012; 24:346-357.

(5) Moody H. (1992) Ethics in an aging society. Baltimore: The Johns Hopkins University Press, 1992.

(6) Denier Y, Dierckx de Casterlé B, De Bal N et al. "It's intense, you know”: nurses' experiences in caring for patients requesting euthanasia. Med Health Care and Philos 2010; $13: 41-48$.

(7) Denier Y, Gastmans C, De Bal N et al. Communication in nursing care for patients requesting euthanasia: a qualitative study. J Clin Nurs 2010; 19:3372-3380.

(8) Ohnsorge K, Gudat Keller H, Widdershoven G et al. 'Ambivalence' at the end of life: how to understand patients' wishes ethically. Nurs Ethics 2012;19. 
(9) Gastmans C, Mahieu L, Vanlaere L et al. Author response: what's wrong with a broad and particularistic perspective on normativity? Nurs Ethics 2011; 18:264-265.

(10) Vanlaere L and Gastmans C. A personalist approach to care ethics. Nurs Ethics 2011; 18:161-173.

(11) Goodin R. (1985) Protecting the vulnerable. A reanalysis of our social responsibilities. Chicago: The University of Chicago Press.

(12) Sellman D. Towards an understanding of nursing as a response to human vulnerability. Nurs Philos 2005; 6:2-10.

(13) Bryon E, Dierckx de Casterlé B, Gastmans C. 'Because we see them naked': nurses' experiences in caring for hospitalized patients with dementia considering artificial nutrition or hydration (ANH). Bioethics 2012; 26:285-295.

(14) Dinc L, Gastmans C. Trust and trustworthiness in nursing. An argument-based literature review. Nurs Inquiry 2012; 19:223-237.

(15) Walker M. (2003) Moral understandings. A Feminist study in ethics. New York: Routledge.

(16) Tronto J. (1993) Moral boundaries: a political argument for an ethic of care. New York: Routledge.

(17) Gastmans C, Dierckx de Casterlé B, Schotsmans P. Nursing considered as moral practice: a philosophical-ethical interpretation of nursing. Kennedy Inst Ethics 1998; 8:43-69.

(18) Chochinov H. Dignity and the essence of medicine: the A,B,C and D of dignity conserving care. BMJ 2007; 335:184-187. 